\title{
Replicative fitness of historical and recent HIV-1 isolates suggests HIV-1 attenuation over time
}

\author{
Kevin K. Ariën ${ }^{a}$, Ryan M. Troyer ${ }^{b}$, Youssef Galia, \\ Robert L. Colebunders ${ }^{c}$, Eric J. Arts ${ }^{b}$ and Guido Vanham ${ }^{a, d}$
}

\begin{abstract}
Background: Changes in virulence during an epidemic are common among pathogens, but still unexplored in the case of HIV-1. Here we used primary human cells to study the replicative fitness of primary HIV-1 isolates from untreated patients, comparing historical (1986-1989) and recent samples (2002-2003).

Methods: Head-to-head dual virus infection/competition assays were performed in both peripheral blood mononuclear cells and human dendritic cell/T-cell co-cultures with pairs of 12 carefully matched historical and recent HIV-1 isolates from untreated patients. Sensitivity to inhibition by lamivudine (3TC) and TAK-779 of historical and recent R5 HIV-1 isolates was measured in a subset of samples.

Results: Overall, the historical HIV-1 out-competed the recent HIV-1 isolates in 176 of 238 competitions and in 9 of 12 competitions carefully matched for CD4 cell count. The mean relative replicative fitness (W) of all historical HIV-1 strains was significantly greater than that of recent HIV-1 isolates $\left(W_{1986-1989}=1.395\right.$ and $W_{2002-2003}=0.545$, $P<0.001$ (t test)). The more fit viruses (mean $\mathrm{W}>1$ ) from 1986-1989 appeared less sensitive to TAK-779 and 3TC than did the less fit (mean $W<1$ ) 2002-2003 viruses.

Conclusions: These findings suggest that HIV-1 replicative fitness may have decreased in the human population since the start of the pandemic. This 'attenuation' could be the consequence of serial bottlenecks during transmission and result in adaptation of HIV-1 to the human host. (C) 2005 Lippincott Williams \& Wilkins
\end{abstract}

AIDS 2005, 19:1555-1564

Keywords: replication capacity, fitness, evolution, attenuation, HIV-1 epidemic

\section{Introduction}

Zoonotic jumps from SIV-infected chimpanzees and sooty mangabeys are the likely origin of HIV-1 and HIV2 (respectively) in humans [1]. HIV-2 appears less pathogenic than HIV-1 [2,3] and, although some rare cases of infections with defective HIV-1 have been documented [4-6], the vast majority of HIV-1 infections results in lethal immunodeficiency disease in man. Within the simian hosts, infection with these native SIVs do not manifest into severe clinical symptoms whereas experimental transfers of SIV into non-native simian hosts (such as macaques) usually results in a rapid and fatal outcome $[7,8]$.

Molecular adaptation and continuous selection may influence HIV virulence in the human population $[9,10]$. Although current theories suggest that HIV-1 replicative fitness and genetic diversity increases during disease progression [11-13] in the face of host immune pressure, the stringent genetic bottlenecks upon transmission may in effect reset the fitness to a lower set-point in each new host. As with decreasing virulence with other lethal pathogens in a host population [14], HIV-1 may begin to

From the a Department of Microbiology, HIV and Retrovirology Research Unit, Institute of Tropical Medicine, Antwerp, Belgium, the ${ }^{b}$ Division of Infectious Diseases, Department of Medicine, Case Western Reserve University, Cleveland, Ohio, USA, the ${ }^{\mathrm{c}}$ Department of Clinical Sciences, Institute of Tropical Medicine, Antwerp, Belgium, and the ${ }^{\mathrm{d} D e p a r t m e n t}$ of Biomedical Sciences, Faculty of Pharmaceutical, Veterinary and Biomedical Sciences, University of Antwerp, Belgium.

Correspondence to K. K. Ariën, Department of Microbiology, HIV \& Retrovirology Research Unit, Institute of Tropical Medicine, Nationalestraat 155, B-2000 Antwerp, Belgium.

Tel: +32 3247 6489; fax: +32 3247 6333; e-mail: karien@itg.be

Received: 4 May 2005; revised: 22 June 2005; accepted: 11 July 2005. 
attenuate in an epidemic if the viral fitness set-point during a new infection is frequently lower than the fitness gain during disease. Virulent viruses that have fast rates of killing, also have a reduced transmission opportunity $\left(\mathrm{R}_{0}\right)$. Thus, lethal viruses with lower virulence may be selected in the human population $[14,15]$. Although the 'attenuation' hypothesis has been tested for HIV-1 in vitro [16], evolution and replicative fitness of HIV-1 during the human epidemic has not been studied, partly due to limited availability of well-documented primary HIV-1 isolates from the early epidemic (1980s).

Most research studies on HIV-1 replicative fitness have established that drug resistant mutations reduce replication capacity $[17,18]$ in the absence of antiretroviral drugs. However, HIV-1 replicative fitness actually increases during disease progression in treatment-naive patients $[11,12,19]$. We have recently defined two parameters of ex vivo fitness: 'pathogenic fitness' which measures the relative HIV-1 replicative fitness in primary human $\mathrm{T}$ cells, i.e., the major target cells during chronic HIV-1 infection; and 'transmission fitness' which measures the efficiency of viral transfer from dendritic cells (i.e., crucial targets during sexual HIV-1 transmission) to T cells [2024]. In this study we have compared the pathogenic and transmission fitness of primary HIV-1 isolates from the beginning of the epidemic (1986-1989) and more recent viruses (2002-2003) from the same clinic in Antwerp, Belgium. Direct head-to-head competitions of HIV-1 pairs (almost every combination) in peripheral blood mononuclear cells (PBMC) or interstitial-type like dendritic cells/T-cell cocultures suggest diminished fitness of the more recent HIV-1 isolates. Recent findings suggest that in the absence of drug resistance mutations, reduced HIV-1 fitness is attributable to decreased efficiency of host cell entry and maps to the HIV-1 env gene. Evidence that the more recent HIV-1 isolates were more sensitive to a CCR5-antagonist (TAK-779) supports the hypothesis of reduced entry efficiency $[23,25]$.

\section{Methods}

\section{Cells}

PBMC, obtained from a HIV-seronegative buffy coat by Ficoll-Hypaque density gradient centrifugation, were stimulated with $2 \mu \mathrm{g} / \mathrm{ml}$ phytohemagglutinin (PHA) for 3 days and further maintained in RPMI 1640-2 mM L-glutamine supplemented with $10 \%$ fetal bovine serum, $10 \mathrm{mM}$ HEPES, $1 \mathrm{ng} / \mathrm{ml}$ interleukin-2 (IL-2), $100 \mathrm{U} / \mathrm{ml}$ penicillin and $100 \mu \mathrm{g} / \mathrm{ml}$ streptomycin. Monocytederived dendritic cells (MO-DC) were generated as previously described [20]. Frozen lymphocyte fractions were thawed on the day of infection and used to isolate autologous CD4 T cells.

\section{Patients and viruses}

Twenty-four patients were selected from our cohort at the Institute of Tropical Medicine in Antwerp, Belgium. Twelve historical samples from untreated subjects in 1986-1989 were selected based on availability of primary patient cells (PBMC) and plasma (continuously stored in liquid nitrogen and at $-80^{\circ} \mathrm{C}$, respectively). In addition, we collected PBMC and plasma from 50 untreated patients during 2002-2003 with available clinical/ immunological data and stored the samples accordingly until use. Patient samples were further characterized for genetic subtype (Env and Pro-RT), plasma bDNA viral load and co-receptor tropism (as determined on U87.CD4 cells expressing either CCR 5 or CXCR4). Subsequently, 12 viruses isolated in 2002-2003 were matched with the historical samples for CD4 cell count, co-receptor tropism and genetic subtype. Both groups of drug-naive patients were not significantly different for CD 4 cell count $(P=0.67$, paired t test $)$ and viral load $(P=0.40$, paired t test). Overall, 16 HIV-1 isolates were obtained from patients in early disease (i.e., CD4 cell counts typically $>400 / \mu$ land non-syncytium inducing/ CCR 5 tropic), while eight viruses were isolated from patients in advanced disease (i.e., CD4 cell counts typically $<200 / \mu 1$ and syncytium-inducing/CXCR 4 tropic (Fig. 1a). Virus stocks were propagated from primary patient cells in short-term cocultures with HIV-seronegative donor PBMC. Subsequently, tissue culture dose for $50 \%$ infectivity $\left(\right.$ TCID $_{50}$ ) was calculated using the Reed and Muench method [26]. Briefly, all virus stocks were serially diluted in sixfold and then added to activated PBMC. Following 12 days of incubation, supernatant was harvested and virus production was measured using an in-house p24 antigen capture ELISA [27]. Finally, 12 matched pairs of viruses were used in dual virus competition assays to measure relative fitness.

\section{Growth competition assays}

Nearly 750 competitions were performed with 24 HIV-1 primary isolates using PHA/IL-2 activated PBMC from one donor (same donor as for $\mathrm{TCID}_{50}$ determination and same blood draw) and in duplicate. A subset of competitions (five virus pairs) was repeated in PBMC from an additional donor to exclude host effects. Finally, the same NSI/R5 isolates were used in dual infections with MO-DC and autologous non-activated CD4 $\mathrm{T}$ cells. Cells were infected with two viruses at equal multiplicity of infection (0.0005 infectious units/cell for PBMC and 0.001 infectious units/cell for MO-DC), uninfected cultures were used as HIV-negative controls and monoinfected cell cultures of each virus correspond to positive controls. As described elsewhere $[11,12,23,24]$, virus mixtures were incubated with $2 \times 10^{5} \mathrm{PBMC}$ or $1 \times 10^{5} \mathrm{MO}-\mathrm{DC}$ at $37^{\circ} \mathrm{C}$ in $5 \%$ $\mathrm{CO}_{2}$ and washed three times with $1 \times$ phosphatebuffered saline $24 \mathrm{~h}$ post-infection and then resuspended in complete medium. Subsequently, $3 \times 10^{5}$ autologous 


\begin{tabular}{|c|c|c|c|c|c|c|c|c|c|}
\hline Virus name & Subtype & $\begin{array}{l}\text { Co-receptor } \\
\text { tropism }\end{array}$ & $\begin{array}{l}\text { CD4 count } \\
\text { (cells/ } / \mu \mathrm{L} \text { ) }\end{array}$ & $\begin{array}{c}\text { Viral load } \\
\text { (log10 copies } / \mathrm{mL} \text { ) }\end{array}$ & Virus name & Subtype & $\begin{array}{l}\text { Co-receptor } \\
\text { tropism }\end{array}$ & $\begin{array}{l}\text { CD4 count } \\
\text { (cells/ } / \mathrm{L} \text { ) }\end{array}$ & $\begin{array}{c}\text { Viral load } \\
\text { (log10 copies } / \mathrm{mL} \text { ) }\end{array}$ \\
\hline VI 64 & F1 & CXCR4 & 40 & 4.31 & VI 342 & B & CCR5 & 530 & 4.04 \\
\hline VI 2617 & 01_AE & CXCR4 & 23 & 4.51 & VI 2622 & B & CCR5 & 522 & 4.58 \\
\hline VI 75 & $\mathrm{~A} 1$ & CCR5 & 120 & 3.84 & VI 178 & 05_DF & CCR5 & 570 & 4.28 \\
\hline VI 2621 & 01_AE & CCR5/CXCR4 & 198 & 4.95 & VI 2641 & 02_AG & CCR5 & 524 & 4.90 \\
\hline VI 218 & B & CCR5/CXCR4 & 190 & 5.54 & VI 48 & $G$ & CCR5 & 622 & 3.66 \\
\hline VI 2678 & B & CCR5 & 214 & 4.42 & VI 2642 & 02_AG & CCR5 & 607 & 3.81 \\
\hline VI 339 & $B$ & CCR5 & 260 & 3.25 & VI 276 & B & CCR5 & 650 & 4.39 \\
\hline VI 2626 & $\mathrm{~B}$ & CCR5 & 215 & 5.30 & VI 2627 & 14_BG & CCR5 & 654 & 4.28 \\
\hline VI 180 & $B$ & CCR5 & 400 & 4.26 & VI 318 & $\mathrm{~B}$ & CCR5 & 760 & $n d$ \\
\hline VI 2673 & $\mathrm{~B}$ & CCR5 & 409 & 4.27 & VI 2681 & B & CCR5 & 714 & 3.71 \\
\hline VI 321 & $B$ & CXCR4 & 430 & 5.24 & VI 334 & B & CCR5/CXCR4 & 940 & 3.98 \\
\hline VI 2676 & B & CCR5 & 430 & 4.40 & VI 2687 & B & CCR5 & 1086 & 3.89 \\
\hline
\end{tabular}

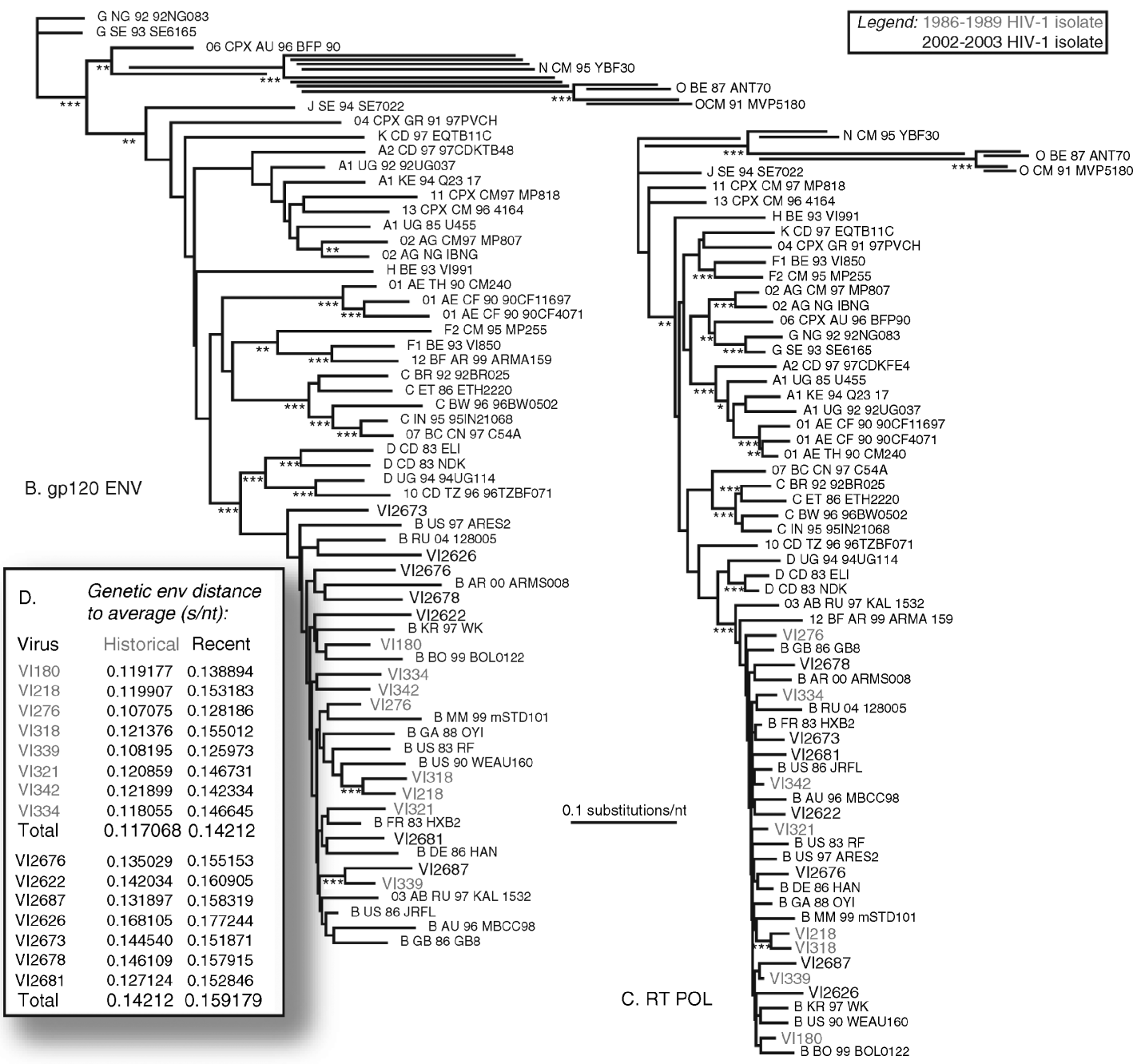

Fig. 1. Virus characteristics (a) and phylogenetic analyses of historical (1986-1989) and more recent (2002-2003) HIV-1 subtype B isolates based on Env V1-V5 (b) and Pol (b) sequences. (a) Virus and patient characteristics of the HIV-1 isolates used (1986-1989 viruses are in grey, 2002-2003 viruses are in black). Sequences were aligned with Clustal X and then manually edited with BioEdit using the amino acid sequence for misaligned insertions-deletions. All sequence gaps were stripped from the alignment and phylogenetic trees were built with PAUP*4.0b10. Neighbour-joining trees were constructed by using the HKY85 model of nucleotide substitution, with the transition/transversion ratio and alpha shape parameter for a gamma distribution estimated directly from the data under maximum likelihood. The resulting model was used in a heuristic search with the subtree pruning-regrafting algorithm for branch swapping to find the optimum tree. The significance of the branching order was estimated by bootstrap resampling of 1000 replicates. Bootstrap values are displayed on significant branches $\left(^{* * *}>900 ;{ }^{* *}>800,{ }^{*}>700\right)$. Historical (1986-1989) and more recent isolates (2002-2003) analysed in this study are displayed on the trees in grey and black, respectively (b and c). Average env genetic distances $(\mathrm{d})$ were calculated according to the Kimura 2-parameter model using MEGA 2.0 [44]. GenBank sequence accession numbers: DQ177188-DQ177211 (Env) and DQ177212-DQ177234 (Pol). 
CD4 T cells were added to MO-DC cultures and fed with complete medium twice weekly. Cell-free supernatant was assayed for p24 antigen detection 4 and 7 days postinfection. Two aliquots of supernatants and cells were harvested at day 10 after infection and stored at $-80^{\circ} \mathrm{C}$ for subsequent analysis.

\section{PCR}

Proviral DNA was extracted from lysed PBMC using the QIAamp DNA Blood kit (Qiagen, Qiagen Benelux b.v., Venlo, The Netherlands). HIV-1 DNA was PCR amplified using a set of external primers (envB: $5^{\prime}$-AGAAAGAGCAGAAGACAGTGGCAATGA$3^{\prime}$ and ED14: 5' - TCTTGCCTGGAGCTGTTTGATGCCCCAGAC - $3^{\prime}$ ) followed by nested amplification (E80: 5'—CCAATTCCCATACATTATTGTG-3' and E125: 5'-CAATTTCTGGGTCCCCTCCTGAGG-3'). Both the external and nested PCR reaction was carried out in a $100-\mu l$ reaction mixture under defined cycling conditions as reported earlier [11].

\section{Heteroduplex tracking assay}

Nested PCR products in env (C2V3) were analysed by heteroduplex tracking assays (HTA) to determine the amount of virus production in the dual infection/ competition experiments, as described previously $[11,12,23,24]$. Radiolabelled DNA probes were amplified from regions of env by PCR using the same primer sets described above, and one of the nested primers was radiolabelled using $\mathrm{T} 4$ polynucleotide kinase and $2 \mu \mathrm{Ci}$ $\left[\gamma-{ }^{32} \mathrm{P}\right] \mathrm{ATP}$. We used the laboratory adapted HIV-1 strains IIIB and Ba-L and two subtype B primary isolates (969-1 and 969-6) to generate radiolabelled DNA probes and the same probes were used consistently to analyse all competitions (subtype B and non-B), both in PBMC and DC/CD4 T-cell cultures. Each competition was analysed, separately, with at least three different probes. From previous analyses on diverse HIV types (1 and 2), groups (O and $\mathrm{M})$, HIV-1 $\mathrm{M}$ subtypes (A, B, C, D, 01_AE), we know that probe binding and specificity does not differ significantly among HIV-1 M subtypes [11,23,24]. Subsequently, radiolabelled PCR-amplified probes were separated on $1 \%$ agarose gels and purified using the QIAquick gel extraction kit (Qiagen). HTA reaction mixtures containing DNA annealing buffer $(100 \mathrm{mM}$ $\mathrm{NaCl}, 10 \mathrm{mM}$ Tris-HCl pH 7.8, 2 mM EDTA), $10 \mu \mathrm{l}$ amplified DNA from the competition culture, and 0.1 pmol radioactive probe DNA were denatured at $95^{\circ} \mathrm{C}$ for $3 \mathrm{~min}$ followed by incubation at $37^{\circ} \mathrm{C}$ for $5 \mathrm{~min}$ and rapid transfer on wet ice to allow re-annealing. DNA heteroduplexes were resolved on 5\% TBE nondenaturing polyacrylamide gels (Bio-Rad, Bio-Rad Laboratories, Nazareth, Belgium) for $75 \mathrm{~min}$ at $200 \mathrm{~V}$. Gels were dried for $45 \mathrm{~min}$ at $80^{\circ} \mathrm{C}$, exposed and scanned with a phosphor imager (Cyclone, PerkinElmer) and analysed with OptiQuant (PerkinElmer, Zaventem, Belgium).

\section{Estimation of viral fitness}

The final ratio of two viruses produced in a dual infection was estimated by heteroduplex tracking analysis and compared to the production in monoinfections. Production of individual HIV isolates in a dual infection $\left(\mathrm{f}_{\mathrm{o}}\right)$ was divided by the initial proportion in the inoculum $\left(i_{\mathrm{o}}\right)$. This is referred to as relative fitness $\left(W=f_{o} / i_{o}\right)$, while the ratio of the relative fitness values of each HIV variant in the competition is a measure of the fitness difference $\left(\mathrm{W}_{\mathrm{D}}\right)$ or ratio between two HIV strains $\left(\mathrm{W}_{\mathrm{D}}=\mathrm{W}_{\mathrm{M}} /\right.$ $\mathrm{W}_{\mathrm{L}}$ ), with $\mathrm{W}_{\mathrm{M}}$ and $\mathrm{W}_{\mathrm{L}}$ corresponding to the relative fitness of the more and less fit virus, respectively [11].

\section{Sensitivity to TAK-779 and lamivudine}

Sensitivity of NSI/R 5 primary isolates was determined in experiments with CCR 5 co-receptor antagonist TAK779 and non-nucleoside reverse transcriptase (RT) inhibitor lamivudine (3TC) (both obtained from NIH AIDS Research \& Reference Reagent Program). U87.CD4.CCR 5 cells were incubated with serial drug dilutions (0.1 nM-10 $000 \mathrm{nM}$ for both TAK-779 and 3TC) $1 \mathrm{~h}$ prior to infection with HIV-1. Excess drug and virus was washed away and medium containing drug was replaced twice weekly. Drug was present before, during and after infection. At day 10, aliquots of supernatant were analysed for p24 antigen production.

\section{Subtyping and sequence analyses}

Envelope fragments (nucleotides 6594-7774, DQ177188DQ177211) were amplified by PCR and sequenced using external primers envB, 5'-AGAAAGAGCAGAAG ACAGTGGCAATGA-3 - $3^{\prime}$ and ED14, 5'-TCT TGCCTGGAGCTGTTTGATGCCCCAGAC-3' $(1760 \mathrm{nt})$ and internal primers ED5, 5'-ATGGGAT CAAAGCCTAAAGCCATGTG-3' and ED12, 5'-AGTGCTTCCTGCTGCTCCCAAGAACCCA AG-3' $3^{\prime}$ (1255 nt). Pol fragments (nt 2280-3518, DQ177212-DQ177234) corresponding to protease amino acid 10 through RT amino acid 323 were PCR amplified and sequenced using external primers PS1, 5'—TTTTTTAGGGAAAATTTGGCCTTC- - $3^{\prime}$ and RTA9, 5'-TAAATTTAGGAGTCTTTCCCCATA- $3^{\prime}(1633 \mathrm{nt})$ and internal primers PS2, 5'TCCСТCAAATCACTCTTTGGCAAC- $-3^{\prime}$ and RTA6subB, 5'-CCATTGGCCTTGCCCCTGCTT CTG-3' (1310 nt). Nucleotide sequencing was performed using an ABI 3730 DNA sequencer at Davis Sequencing Inc (Davis Sequencing, INC., Davis, California, USA). For phylogenetic subtyping analyses, nucleotide sequences were aligned using ClustalX and then manually edited for codon alignment. Alignments included a representative set of known subtype sequences recommended by the Los Alamos HIV Sequence Database. For env alignment, all sequence gaps were removed (there were no gaps in the pol alignment), neighbour-joining trees were generated using ClustalX with 1000 bootstrap replicates and consensus trees were displayed with TreeView [28]. 


\section{Results}

Characterization of historical and recent HIV-1 isolates

Two sets of 12 HIV-1 isolates were propagated from patient samples collected in 1986-1989 and in 20022003. These patient samples were carefully matched based on CD4 cell count and virus properties (viral load, predicted co-receptor tropism and genetic subtype) (Fig. 1a). Two-thirds of the HIV-1 isolates were NSI/ CCR 5 tropic and obtained from patients in relatively early disease (i.e., CD4 cells typically $>400 \mu \mathrm{l}$ ), while one-third were SI/CXCR4 tropic and isolated from patients in advanced disease (i.e., CD4 cells typically $<200 \mu \mathrm{l}$ ). Infection of U87.CD4.CCR 5 or U87.CD4. CXCR 4 cells was the basis for determining co-receptor usage. Fifteen of 24 viruses were classified as subtype B based on DNA sequencing and phylogenetic analyses of the env gp120 coding region and the protease-RT region of pol. The other nine HIV-1 isolates were of different subtypes (A1, G, F1) and circulating recombinant forms (CRF01_AE, CRF02_AG, CRF05_DF, CRF14_BG). Phylogenetic trees were also constructed to confirm genetic intermixing of both historical and recent subtype $\mathrm{B}$ viruses in both the pol and env genes (Fig. 1b and c). DNA distance matrices indicated that recent subtype B HIV-1 isolates were slightly more heterogeneous in both pol and env genes (Fig. 1d). However, genetic distances between recent and historical HIV-1 isolates were not significantly different. Finally, a PSSM score for all of the V3 sequences of each subtype B isolate confirmed the tropism determined by the U87.CD4.CCR 5 (or CXCR4) infection experiments (see online supplementary data) [29].

\section{Comparing the replicative fitness of historical and recent $\mathrm{HIV}-1$ isolates in PBMC}

HIV-1 isolates of 24 untreated patients were used in full pair wise, head-to-head competitions using activated human PBMC as target cells. Upon peak virus production, viral DNA was PCR amplified and used in a modified HTA to measure dual virus production. The estimated frequency of recombination between HIV-1 isolates in the dual infection competition assay is $0.1 \% / 1000$ base pairs, well below the limit of HTA detection [11]. HTA involves denaturation and annealing of PCR-amplified HIV-1 env products from the dual infection with a radiolabelled $e n v$ probe from a different HIV-1 strain. Heteroduplex bands representing both HIV-1 isolates in the dual infection migrate to distinct positions on a non-denaturing polyacrylamide gel, which is then quantified using phosphorimaging. At least two probes were used for each competition resulting in identical fitness values. We have previously performed a series of HTA controls for PCR amplification, probe annealing, and quantification [11,12,23,24,30,31]. This technique can be used with any two HIV isolates without specific sequence information and was even more reliable, quantitative, and sensitive (up to 500-fold differences in fitness) than real-time PCR [32].

The majority of competitions between historical and recent HIV-1 isolates from patients attending the same clinic in Antwerp showed that recent isolates were less fit than historical viruses (Fig. 2, Fig. 3). Historical HIV-1 out-competed recent viruses in 176 out of 238 competitions (74\%) and the mean relative inter-group replicative fitness (W) (i.e., direct competitions between isolates from both groups) was 1.395 and 0.545 , respectively $(P<0.001$, t test $)$. Direct competitions between isolates within the same group (i.e., intra-group fitness) showed no apparent fitness differences (i.e., $\mathrm{W} \cong 1$ ). The relative replicative fitness value for the $1986-1989$ viruses was $0.959(P=0.85)$ and $\mathrm{W}_{2002-}$ ${ }_{2003}=0.932(P=0.96)$ for the recent HIV-1 (Fig. 3). Twelve competitions within the complete set involved pairs of historical and recent isolates that were derived from patient samples with matched CD4 cell counts, viral load, HIV-1 co-receptor tropism, and virus subtype. Historical patient viruses out competed their 2002-2003 counterparts in nine out of twelve of these competitions (Fig. 2).

To exclude possible influence of divergent HIV-1 subtypes, we re-analysed the data for the 15 subtype B isolates and confirmed that subtype B viruses of the 1980s were significantly more fit than those isolated in 2002$2003\left(\mathrm{~W}_{1986-1989}=1.361\right.$ and $\mathrm{W}_{2002-2003}=0.565$, $P=0.014$, t test) (Fig. 3). A subset of competitions between viruses of the two groups was repeated in PBMC of another donor, to exclude possible host effects. Total HIV-1 production was higher in the PBMC of one donor but the relative production of each virus (i.e., relative replicative fitness) in the dual infection was similar with both donors (as observed in five independent studies) $[11,12,23,24,30]$.

A factor that may have contributed to this possible attenuation is the advent of antiretroviral therapy (ART) in the late 1980s. Although none of these samples were obtained from patients with ongoing treatment, we still screened for drug resistance mutations by sequencing the protease-RT coding regions of pol (see online supplementary data). Surprisingly, the historical VI 321 was the only patient isolate to harbour a single drug resistance mutation, i.e., K70R encoding resistance to zidovudine [17]. The VI 321 HIV-1 isolate was actually more fit that the vast majority of the recent viruses (Fig. 2k, Fig. 3b).

In a previous study, we showed that R 5 viruses of higher fitness appeared to be less sensitive to entry inhibitors such as PSC-RANTES, TAK-779, and T-20 [30]. To confirm and extend this observation, we measured the sensitivity of the historical and recent R 5 HIV-1 isolates to the RT inhibitor, 3TC and the CCR 5 antagonist, TAK-779. In 


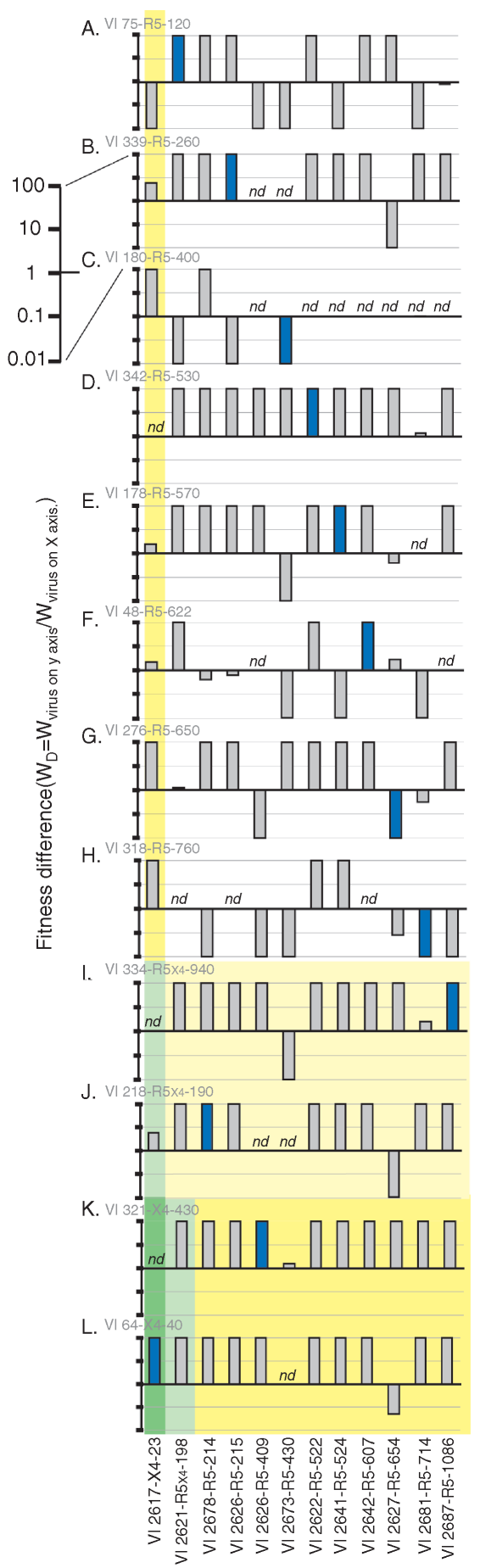

Fig. 2. Comparing the relative replicative fitness difference $\left(\mathrm{W}_{\mathrm{D}}\right)$ of historical and recent $\mathrm{HIV}-1$ patient isolates in direct competition. Historical isolates (VI 75, VI 339, VI 180, VI 342, VI 178, VI 48, VI 276, VI 318, VI 334, VI 218, VI 321, and VI 64) were used to compete against each of the more recent isolates (VI 2617, VI 2621, VI 2678, VI 2626, VI 2673, VI 2622, VI 2641, VI 2642, VI 2627, VI 2681, and VI 2687) with equal multiplicity of infection for each isolate. Each isolate name is followed by the coreceptor tropism and CD4 cell count (e.g., VI 75-R5-120: virus isolate 75 uses CCR5 as coreceptor for entry and has a CD4 cell count of general, the 1986-1989 viruses appeared less sensitive to TAK-779 and 3TC than did the 2002-2003 viruses, but this trend was not statistically significant (Fig. 4a). Viruses characterized as being more fit (mean $\mathrm{W}>1$ ) were also less sensitive to TAK-779 and 3TC than the less fit (mean $\mathrm{W}<1$ ) (Fig. 4b).

\section{Historical HIV-1 isolates may have higher 'transmission fitness'}

Animal models of sexual transmission suggest that dendritic cells may be initial targets of primary HIV-1 infection $[21,22]$. 'Transmission fitness' may be related to the efficiency of virus transfer from dendritic cells to $\mathrm{T}$ cells, whereas the replicative fitness in $\mathrm{T}$ cells may be more related to 'pathogenic fitness' [24]. Based on our observations in PBMC, a subset of the matched historical and recent viruses was competed in autologous dendritic cell/CD4 T-cell co-cultures.

We observed that transfer from dendritic cells to T cells was more efficient for the historical isolates. The two recent HIV-1 isolates (VI 2627 and VI 2681) that were more fit than their historical counterparts (VI 276 and VI 318 ) in PBMC, were also more fit in dendritic cell/CD4 T-cell competitions. In one of these selected pairs where the recent virus out-competed the historical strain (VI 342 vs. VI 2622) in PBMC, dominance of the recent virus (VI 2622) was not absolute since VI 342 had increased 'transmission fitness' in the MO-DC/CD4 T-cell cultures (Fig. 5).

\section{Discussion}

This study provides the first evidence suggesting that present-day HIV-1 patient isolates have reduced replicative fitness as compared to viruses isolated 15 years earlier in the Belgian epidemic. Reduced replicative fitness was shown both in activated human $\mathrm{T}$ cells and in autologous co-cultures of interstitial-like dendritic cells and CD4 T cells (i.e., to model HIV-1 transfer). Finally, we also show that a subset of the same drug-naive 2002-2003 isolates are more sensitive to inhibition by a CCR 5 co-receptor antagonist (TAK-779) and a RT inhibitor (3TC). Our

Fig. 2. (continued)

$120 \mu \mathrm{l})$. Historical and recent HIV-1 strains were further subdivided according to coreceptor tropism: R5 (panel A$\mathrm{H}$ ), R5X4 (panel I and J), and X4 (panel $\mathrm{K}$ and L). All of the X4 versus $X 4$ competitions are grouped in panels $K$ and $L$, the $X 4$ vs. $\mathrm{R} 5 \mathrm{X} 4$ are in panels $\mathrm{I}, \mathrm{J}, \mathrm{K}$, and $\mathrm{L}$, and $\mathrm{R} 5 \mathrm{X} 4$ versus $\mathrm{R} 5$ are in panels I and J. Blue bars represent competitions between historical and recent isolates that were matched for CD4 cell count, coreceptor tropism, bDNA viral load, and genetic subtype. (nd, not done). 

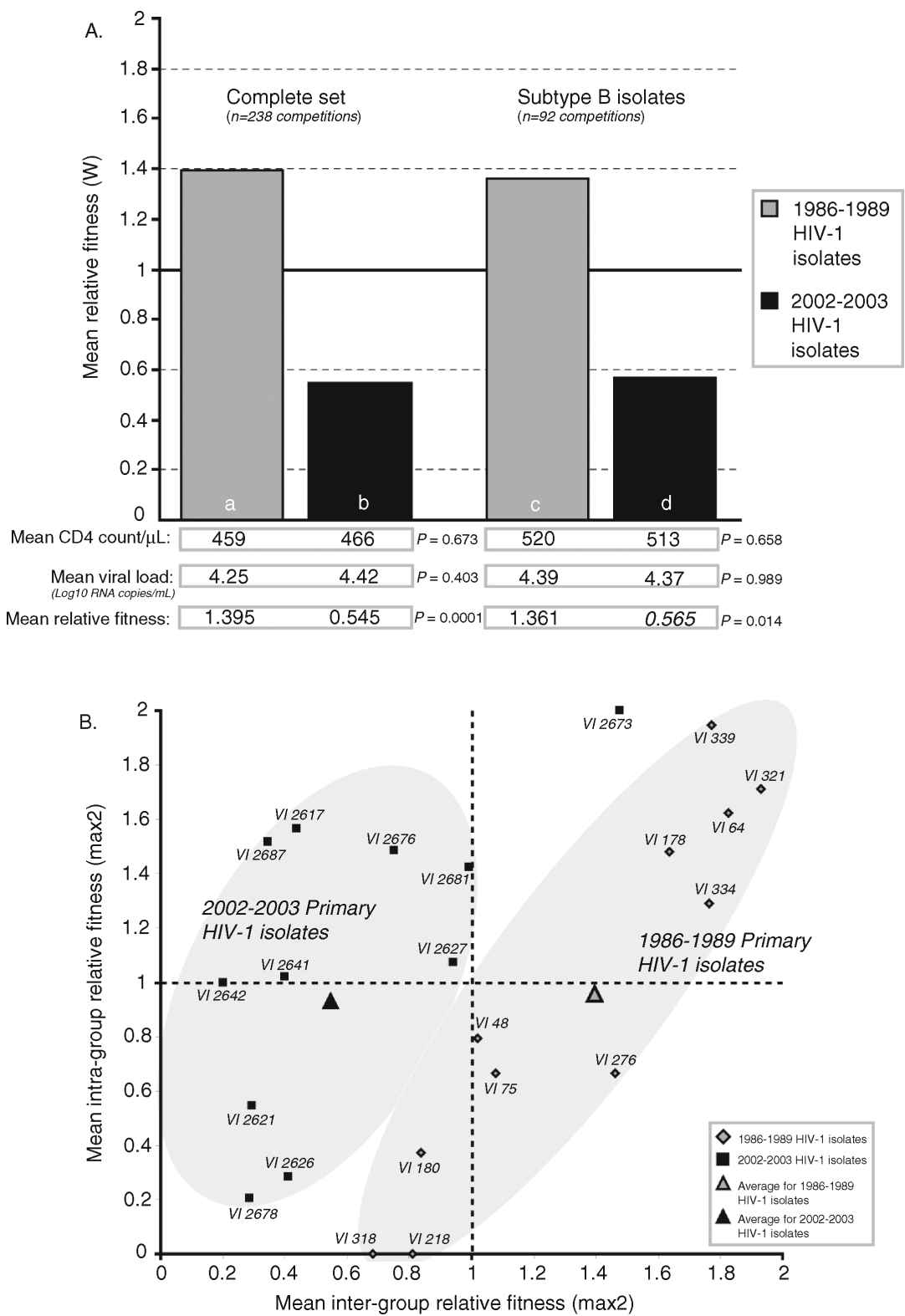

Fig. 3. Mean competitive advantage of historical isolates over recent isolates. (a) Mean replicative fitness of the complete set of dual virus competitions ( $n=238$ competitions) is shown in (a) 1986-1989 isolates and (b) 2002-2003 isolates, while results on subtype B isolates only ( $n=92$ competitions) are shown in (c) 1986-1989 and (d) 2002-2003. Both groups of isolates are not significantly different for CD4 cell count $(P=0.67$ and 0.66 , paired t test) and viral load $(P=0.40$ and 0.99 , paired t test). However, the mean relative fitness between 1986-1989 and 2002-2003 HIV-1 viruses was significantly different in the complete set of competitions involving all 24 viruses $\left(W_{1986-1989}=1.395, W_{2002-2003}=0.545, P<0.001\right)$ and in the subset of subtype $B$ isolates $\left(W_{1986-1989}=1.361, W_{2002-2003}=0.565, P=0.014\right)$. (b) Mean replicative fitness values for inter-group versus intragroup competitions in PBMC. The mean intra-group replicative fitness for 1986-1989 HIV-1 isolates and 2002-2003 HIV-1 isolates is $\mathrm{W}=0.959$ and $\mathrm{W}=0.932$, respectively. However, full pair-wise inter-group replicative fitness comparison revealed that historical isolates are significantly more fit than isolates from 2002-2003. The average inter/intra replicative fitness for each group is represented by the grey and black triangle.

observation on decreased sensitivity to TAK-779 is in line with earlier reports suggesting that differences in fitness may be related to entry efficiency $[23,25,30]$.

Myxoma virus infections in rabbits may be the best documented case of virus-host adaptation and shows that viruses do not per se evolve toward greater pathogenic potential. Introduction of myxoma in the susceptible rabbit population of Australia in 1950 quickly resulted in the outgrowth of attenuated strains [33]. Although difficult to study, epidemio-mathematical models of host-pathogen interaction have suggested that many 

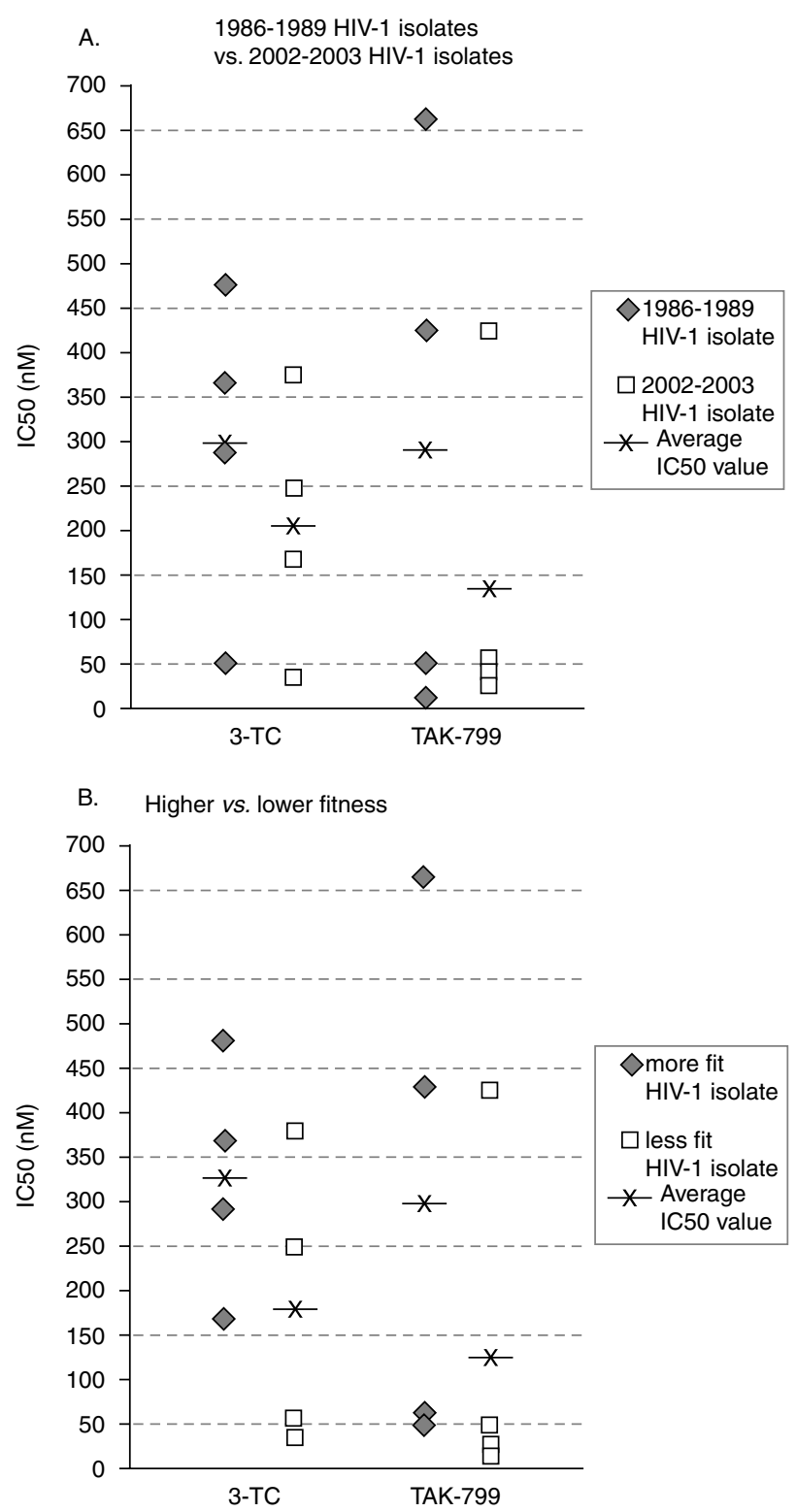

Fig. 4. Sensitivity to inhibition by 3TC and TAK-779. Inhibition experiments with TAK-779 (a CCR5 co-receptor antagonist) and 3TC (a non-nucleoside reverse transcriptase inhibitor) were performed on a representative group of primary HIV-1 isolates. As described in materials and methods, U87.CD4.CCR5 cells were incubated with serial dilutions of both drugs $(0.1 \mathrm{nM}-10000 \mathrm{nM})$ for $1 \mathrm{~h}$ and in sixfold replicates. Virus (0.001 infectious units/cell) was added to pre-treated cells and cultures were incubated for $24 \mathrm{~h}$ at $37{ }^{\circ} \mathrm{C}$ and $5 \% \mathrm{CO}_{2}$. Virus and drug were washed away and cultures were fed twice weekly with drug-containing medium. At 10 day post-infection, aliquots of supernatant were assayed for $\mathrm{p} 24$ antigen concentration. A representative set of eight primary isolates (i.e., four isolates from 19861989 and four from 2002-2003), matched for CD4 cell count and co-receptor tropism, was assayed for sensitivity to TAK779 and 3 TC. The $50 \%$ inhibitory concentration $\left(\mathrm{IC}_{50}\right)(\mathrm{nM})$ obtained for each isolate is shown (diamonds for 1986-1989 isolates and boxes for 2002-2003 isolates). A line displays the lethal viruses and bacteria have evolved to low virulence or even to a symbiotic existence with the host [14]. We recently established a fitness order for most human immunodeficiency lentiviruses: HIV-1 group $\mathrm{M}>\mathrm{HIV}-$ $2>\mathrm{HIV}-1$ group O [24]. Interestingly, HIV-2 was of high prevalence in West Africa during the late 1980s and early 1990s but has precipitously diminished over the last 10 years due to poor transmission and low virulence [3]. Although there may be many host genetic factors affecting HIV-1 virulence, the most striking examples are protection due to the deletion in a CCR 5 allele [34] and slower disease progression due to multiple CCL3L1 (MIP-1 $\alpha \mathrm{P})$ gene copies [35]. However, unlike virus attenuation, selection in human hosts leading to reduced virulence, requires a high HIV-1 prevalence, high transmission rates, frequent opportunity for transmission and a much longer time.

The mechanisms responsible for a possible HIV-1 attenuation are complex and multifaceted but are likely to involve directional evolution towards increased survival and transmission at the cost of lowering virulence. Over the course of nearly three decades HIV-1 has probably evolved and lost replicative fitness to avoid cytotoxic Tlymphocytes (CTL) [36,37] and humoral immune responses as well as ART interventions [38]. Recent studies provide evidence for the adaptation of HIV-1 to HLA-restricted immune responses at the population level [39]. As HIV-1 sweeps through the human population, the human immune system leaves behind host-specific footprints in the viral genome. However, Bonhoeffer et al. [40] described positive epistasis in the HIV-1 subtype B epidemic, which implies that mutation and recombination may benefit for the virus in the human population. Unfortunately, these comparisons of genetic evolution and replicative fitness have been limited to the HIV-1 protease-RT regions derived from patients receiving ART. Our observations are not based on one specific genomic region, but on the complete virus as it is circulating in the drug-naive human population. However a key question remains: does shared CTL responses/ HLA types in specific human populations or various host genetic polymorphisms affect temporal virus evolution and as a consequence, virulence?

Fig. 4. (continued)

average $I C_{50}$ value $(\mathrm{nM})$ for each group of viruses. Differential sensitivities of historical and recent viruses are presented in (a), while (b) shows differential sensitivities of the more fit and the less fit viruses. The historical viruses (a) appear to have higher $\mathrm{IC}_{50}$ values $(\mathrm{nM})$, meaning that they are less susceptible to inhibition by both TAK-779 and 3TC. These results suggest that the viruses from 2002-2003, which display poor replication capacity in comparison with isolates from 1986-1989, are more sensitive to inhibition by TAK-779 and 3TC. Similarly, high fitness viruses (b) appear less susceptible to inhibition by TAK-779 and 3TC. 


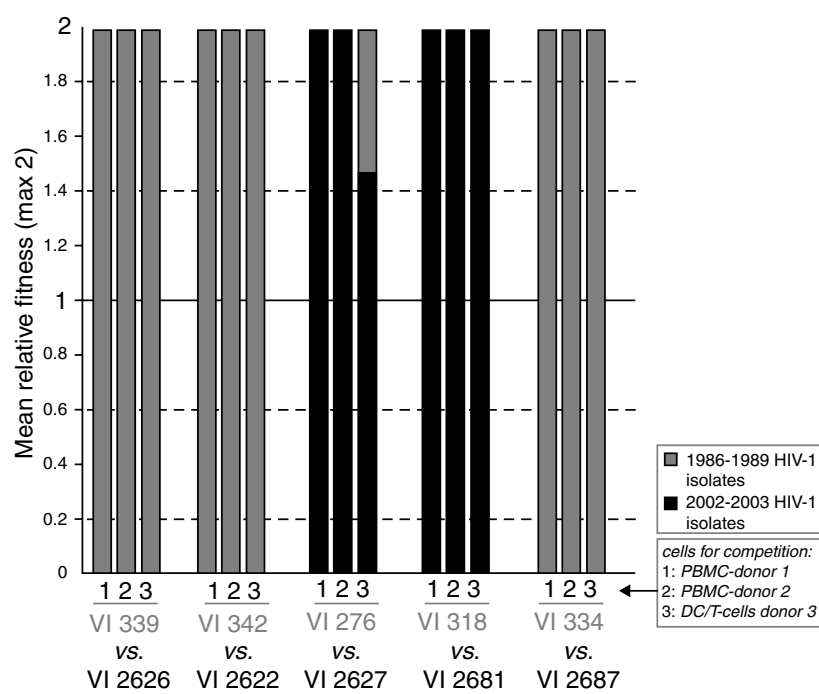

Fig. 5. Host effects and 'transmission fitness'. Relative replicative fitness values derived from pair-wise competitions with NSI/R5 HIV-1 isolates from 1986-1989 and from 2002 2003 that are matched for CD4 T-cell count and viral load. Bars 1 and 2 show results in PBMC of two different human donors, while bar 3 shows results obtained in DC/CD4 T-cell cultures.

Several studies on intra-patient HIV-1 replicative fitness evolution have provided evidence that the replicative fitness of HIV-1 tends to progressively increase through the course of infection in the absence of ART $[11,12,19]$. It is not unlikely that fitness gains that correlate with increasing intra-patient HIV-1 diversity may be host limited due to significant bottlenecking that results from HIV-1 transmission. Consecutive genetic bottlenecks with each transmission event may reset the fitness set point for each new infecting virus. If the reduction in replicative fitness upon transmission is on average greater than the replicative fitness increase within an infected individual prior to new transmission, HIV-1 could attenuate in the human population over time. It is well known that successive transmission events of RNA viruses may cause deleterious genetic bottlenecks that result in profound fitness losses (i.e., Muller's ratchet) [41-43]. As described above, this process of HIV-1 attenuation through genetic bottlenecks at transmission, could only accelerate by ongoing adaptation to common HLA types in the population.

In conclusion, we have studied the evolution of HIV-1 replicative fitness and drug-inhibition efficiency of HIV-1 isolates from untreated patients and show the first experimental evidence suggestive for HIV-1 attenuation over time. Although our observation is based on a limited number of HIV-1 isolates and needs to be confirmed in independent studies, we believe that these findings provide a new paradigm and are crucial in our thinking on HIV-1 evolution in the epidemic.

\section{Acknowledgements}

We are endebted to Valerie De Vos and Betty Willems for assistance with virus isolation, to Katrien Fransen from the AIDS Reference Laboratory in Antwerp and Dr. Marc Van Ranst from the AIDS Reference Laboratory in Leuven for assistance with viral load and to Randall Krizan for assistance with nucleotide sequencing.

Special thanks go the patients attending the AIDS clinic at ITM-Antwerp.

Sponsorship: K.K.A. is supported by a research grant from the Fund for Scientific Research, Flanders (G.0431.02). R.M.T. is supported by an amfAR postdoctoral fellowship (106532-35-RFGN). E.J.A. is supported by research grants from the NIAID, NIH (Al49170, Al57005, and Al43645-02) and from NICHD, NIH (HD-0-3310-502-02).

\section{References}

1. Gao F, Bailes E, Robertson DL, Chen Y, Rodenburg CM, Michael SF, et al. Origin of HIV-1 in the chimpanzee Pan troglodytes troglodytes. Nature 1999; 397:436-441.

2. Kanki PJ, Travers KU, MBoup S, Hsieh CC, Marlink RG, GueyeNDiaye $A$, et al. Slower heterosexual spread of HIV-2 than HIV-1. Lancet 1994; 343:943-946.

3. Gilbert PB, McKeague IW, Eisen G, Mullins C, Gueye-NDiaye A, Mboup S, et al. Comparison of HIV-1 and HIV-2 infectivity from a prospective cohort study in Senegal. Stat Med 2003; 22:573-593.

4. Baltimore D. Lessons from people with nonprogressive HIV infection. N Engl J Med 1995; 332:259-260.

5. Collins $\mathrm{KL}, \mathrm{Nabel} \mathrm{G}$. Naturally attenuated HIV-lessons for AIDS vaccines and treatment. $N$ Engl / Med 1999; 340: 1756-1757.

6. Learmont JC, Geczy AF, Mills J, Ashton LJ, Raynes-Greenow $\mathrm{CH}$, Garsia $\mathrm{RJ}$, et al. Immunologic and virologic status after 14 to 18 years of infection with an attenuated strain of HIV-1. A report from the Sydney Blood Bank Cohort. N Engl J Med 1999; 340:1715-1722.

7. Norley S, Beer B, Holzammer S, zur Megede J, Kurth R. Why are the natural hosts of SIV resistant to AIDS? Immunol Lett 1999; 66:47-52.

8. Rey-Cuille MA, Berthier IL, Bomsel-Demontoy MC, Chaduc Y Montagnier L, Hovanessian AG, et al. Simian immunodeficiency virus replicates to high levels in sooty mangabeys without inducing disease. / Virol 1998; 72:3872-3886.

9. Malim MH, Emerman M. HIV-1 sequence variation: drift, shift, and attenuation. Cell 2001; 104:469-472.

10. Domingo $E$, Holland JJ. RNA virus mutations and fitness for survival. Annu Rev Microbiol 1997; 51:151-178.

11. Quinones-Mateu ME, Ball SC, Marozsan AJ, Torre VS, Albright $J L, V a n h a m G$, et al. A dual infection/competition assay shows a correlation between ex vivo human immunodeficiency virus type 1 fitness and disease progression. J Virol 2000; 74:92229233.

12. Troyer RM, Collins KR, Abraha A, Fraundorf E, Moore DM, Krizan RW, et al. Changes in human immunodeficiency virus type 1 fitness and genetic diversity during disease progression. J Virol 2005. 79:9006-9018.

13. Shankarappa R, Margolick JB, Gange SJ, Rodrigo AG, Upchurch D, Farzadegan $\mathrm{H}$, et al. Consistent viral evolutionary changes associated with the progression of human immunodeficiency virus type 1 infection. / Virol 1999; 73:1048910502. 
14. May RM, Anderson RM. Parasite-host coevolution. Parasitology 1990; 100 (Suppl):S89-S101.

15. Clark SJ, Shaw GM. The acute retroviral syndrome and the pathogenesis of HIV-1 infection. Semin Immunol 1993; 5:149155.

16. Yuste E, Sanchez-Palomino S, Casado C, Domingo E, LopezGalindez C. Drastic fitness loss in human immunodeficiency virus type 1 upon serial bottleneck events. J Virol 1999; 73:2745-2751.

17. Harrigan PR, Bloor S, Larder BA. Relative replicative fitness of zidovudine-resistant human immunodeficiency virus type 1 isolates in vitro. J Virol 1998; 72:3773-3778.

18. Martinez-Picado J, Savara AV, Sutton L, D'Aquila RT. Replicative fitness of protease inhibitor-resistant mutants of human immunodeficiency virus type 1. J Virol 1999; 73:3744-3752.

19. Barbour JD, Hecht FM, Wrin T, Segal MR, Ramstead CA, Liegler $\mathrm{TJ}$, et al. Higher CD4+ T cell counts associated with low viral pol replication capacity among treatment-naive adults in early HIV-1 infection. J Infect Dis 2004; 190:251-256.

20. Vanham G, Penne L, Allemeersch H, Kestens L, Willems B, van der Groen $G$, et al. Modeling HIV transfer between dendritic cells and T cells: importance of HIV phenotype, dendritic cell-T cell contact and T-cell activation. AIDS 2000; 14:2299-2311.

21. Blauvelt A, Asada H, Saville MW, Klaus-Kovtun V, Altman DJ Yarchoan $R$, et al. Productive infection of dendritic cells by HIV-1 and their ability to capture virus are mediated through separate pathways. J Clin Invest 1997; 100:2043-2053.

22. Sivard P, Berlier W, Picard B, Sabido O, Genin C, Misery L. HIV1 infection of Langerhans cells in a reconstructed vaginal mucosa. / Infect Dis 2004; 190:227-235.

23. Ball SC, Abraha A, Collins KR, Marozsan AJ, Baird H, QuinonesMateu ME, et al. Comparing the ex vivo fitness of CCR5-tropic human immunodeficiency virus type 1 isolates of subtypes $B$ and C. / Virol 2003; 77:1021-1038.

24. Ariën KK, Abraha A, Quinones-Mateu ME, Kestens L, Vanham $G$, Arts EJ. The replicative fitness of primary human immunodeficiency virus type 1 (HIV-1) group $M$, group $\mathrm{O}$ and HIV-2 isolates. / Virol 2005. 79:8979-8990.

25. Rangel HR, Weber J, Chakraborty B, Gutierrez A, Marotta ML, Mirza $M$, et al. Role of the human immunodeficiency virus type 1 envelope gene in viral fitness. / Virol 2003; 77:9069-9073.

26. Reed LJ, Muench $\mathrm{H}$. A simple method of estimating fifty per cent endpoints. Am J Hyg 1938; 27:493-497.

27. Beirnaert $E$, Willems B, Peeters $M$, Bouckaert A, Heyndrickx L Zhong $\mathrm{P}$, et al. Design and evaluation of an in-house HIV-1 (group $M$ and $O$ ), SIVmnd and SIVcpz antigen capture assay. JVirol Methods 1998; 73:65-70.

28. Page RD. TreeView: an application to display phylogenetic trees on personal computers. Comput Appl Biosci 1996; 12 357-358.

29. Jensen MA, Li FS, van 't Wout AB, Nickle DC, Shriner D, He HX et al. Improved coreceptor usage prediction and genotypic monitoring of R5-to-X4 transition by motif analysis of human immunodeficiency virus type 1 env V3 loop sequences. J Virol $2003 ; 77: 13376-13388$.
30. Marozsan AJ, Moore DM, Lobritz MA, Fraundorf E, Abraha A, Reeves JD, et al. Differences in the fitness of two diverse wildtype HIV-1 isolates are related to the efficiency of cell binding and entry. I Virol 2005; 79:7121-7134.

31. Marozsan AJ, Fraundorf E, Abraha A, Baird H, Moore D, Troyer $\mathrm{R}$, et al. Relationships between infectious titer, capsid protein levels, and reverse transcriptase activities of diverse human immunodeficiency virus type 1 isolates. / Virol 2004; 78: 11130-11141.

32. Weber J, Chakraborty B, Weberova J, Miller MD, QuinonesMateu ME. Diminished replicative fitness of primary human immunodeficiency virus type 1 isolates harboring the K65R mutation. J Clin Microbiol 2005; 43:13951400.

33. Kerr PJ, Best SM. Myxoma virus in rabbits. Rev Sci Tech 1998; 17:256-268.

34. Dean $M$, Carrington $M$, Winkler C, Huttley GA, Smith MW, Allikmets $R$, et al. Genetic restriction of HIV-1 infection and progression to AIDS by a deletion allele of the CKR5 structural gene. Hemophilia Growth and Development Study, Multicenter AIDS Cohort Study, Multicenter Hemophilia Cohort Study, San Francisco City Cohort, ALIVE Study. Science 1996; 273: 1856-1862.

35. Gonzalez E, Kulkarni H, Bolivar H, Mangano A, Sanchez R, Catano $G$, et al. The influence of CCL3L1 gene-containing segmental duplications on HIV-1/AIDS susceptibility. Science 2005; 307:1434-1440.

36. Leslie AJ, Pfafferott KJ, Chetty P, Draenert R, Addo MM, Feeney $M$, et al. HIV evolution: CTL escape mutation and reversion after transmission. Nat Med 2004; 10:282-289.

37. Friedrich TC, Dodds EJ, Yant LJ, Vojnov L, Rudersdorf R, Cullen $C$, et al. Reversion of CTL escape-variant immunodeficiency viruses in vivo. Nat Med 2004; 10:275-281.

38. Stoddart CA, Liegler TJ, Mammano F, Linquist-Stepps VD, Hayden MS, Deeks SG, et al. Impaired replication of protease inhibitor-resistant HIV-1 in human thymus. Nat Med 2001; 7:712-718.

39. Brander C, Walker BD. Gradual adaptation of HIV to human host populations: good or bad news? Nat Med 2003; 9:13591362.

40. Bonhoeffer S, Chappey C, Parkin NT, Whitcomb JM, Petropoulos CJ. Evidence for positive epistasis in HIV-1. Science 2004; 306:1547-1550.

41. Muller $\mathrm{HI}$. The relation of recombination to mutational advance. Mutat Res 1964; 106:2-9.

42. Escarmis C, Davila M, Charpentier N, Bracho A, Moya A, Domingo E. Genetic lesions associated with Muller's ratchet in an RNA virus. I Mol Biol 1996; 264:255-267.

43. Duarte E, Clarke D, Moya A, Domingo E, Holland J. Rapid fitness losses in mammalian RNA virus clones due to Muller's ratchet. Proc Natl Acad Sci USA 1992; 89:60156019.

44. Kumar S, Tamura K, Jakobsen IB, Nei M. MEGA2: molecular evolutionary genetics analysis software. Bioinformatics 2001; 17:1244-1245. 